\title{
Droplets interaction effects on the heat removed by droplets impact onto heated wall in a polydispersed flow film boiling
}

\author{
J.E. Luna Valencia*1,2, A. V. S. Oliveira², A. Labergue², T. Glantz ${ }^{1}$, M. Gradeck ${ }^{2}$ \\ ${ }^{1}$ IRSN, LEMC B.P. 3, 13115 Saint Paul-Lez-Durance, France \\ ${ }^{2}$ Université de Lorraine, CNRS, LEMTA, F-54000 Nancy, France \\ *Corresponding author email: juan-esteban.luna@irsn.fr
}

\begin{abstract}
A dispersed flow of steam and droplets is formed inside the core of a pressurized water reactor after a loss of primary coolant accident (LOCA). This flow is essential for cooling the core in a LOCA transient. In a previous study, we presented NECTAR, a mechanistic code that calculates heat and mass transfer of a dispersed flow inside a fuel subchannel. This code was validated using experimental results obtained with a test bench named COLIBRI at low droplets' volume fraction $\left(\sim 10^{-4}\right)$. With NECTAR, it was possible to determine that the heat removed by the impact of the drops on the wall is substantial. However, at higher droplet volume fractions, droplets interaction may occur and affect the calculation implemented in NECTAR. Therefore, this work presents numerical simulations of heat transfer by droplets impact using NECTAR for a straight vertical tube with different droplets' volume fraction, from $10^{-4}$ to 0.6 , considering a wetted wall correction factor proposed by Breitenbach et al. [2]. This parameter was added to Gradeck et al. [3] for calculation of impinging droplet heat transfer. We observed negligible interaction for LOCA typical droplets' volume fraction. These simulation results will be validated with experiments to be performed soon in COLIBRI experimental apparatus.
\end{abstract}

\section{Keywords}

NECTAR, mechanistic code, disperse flow, droplet impact, thermal-hydraulics.

\section{Introduction}

During a hypothetical loss of coolant accident (LOCA) in a pressurized water reactor, water is injected into the reactor core to cool it down, a process known as reflooding. During this process, dispersed flow film boiling (DFFB) is created downstream of the quenching front, due to the contact of the injected water with nuclear high-temperature rods. This flow of steam and droplets plays a very important role in cooling the section of fuel rods still not immersed in water. For this reason, the understanding of this phenomenon is important in order to ensure nuclear safety. However, the characterization of this two-phase flow is complex, due to the different physical phenomena involved, such as turbulence, break-up, and droplets coalescence, the dynamic and thermal interaction between the phases, and the thermodynamic non-equilibrium between the phases. The different physical phenomena involved downstream of the quenching front are represented in Fig. 1.

The typical values of the characteristics parameters of this flow are described in Table 1 . The predominant mechanism in the cooling of the fuel rods by the steam/droplets flow is the steamto-wall convection. Nevertheless, cooling given by the impact of droplets on the wall is not negligible [1]. Moreover, the fluid area in the sub-channels could be severely reduced by the ballooning of fuel rods during the LOCA transient. Thus, the amount of steam entering the subchannels is reduced because of the deviation of the steam flow into less blockage subchannels. In these conditions, droplets' impacts onto the wall could play a major role in the blocked subchannel cooling.

Contributions of convective, radiative, and dry contact heat transfer mechanisms depend on local conditions in DFFB, due to the mechanical and thermal non-equilibrium [4]. As a result, 


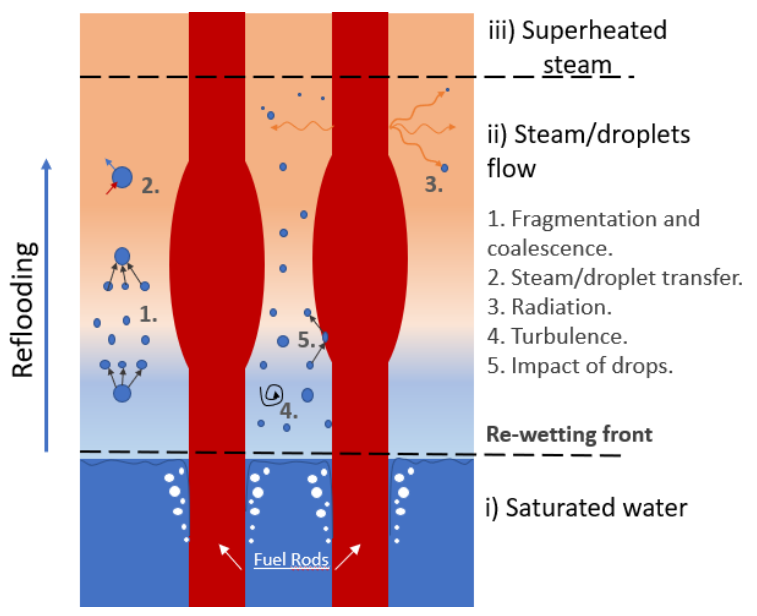

Figure 1. Heat and mass transfer phenomena in LOCA. ([1]).

Table 1. Typical values during LOCA of the dispersed flow.

\begin{tabular}{c|c}
\hline Parameters & Typical LOCA values \\
\hline Droplet diameter $(d)$ & $50 \mu \mathrm{m}-1300 \mu \mathrm{m}$ \\
Axial droplet velocity $\left(u_{d}\right)$ & $4 \mathrm{~m} / \mathrm{s}-16 \mathrm{~m} / \mathrm{s}$ \\
Volume fraction of droplets $(\alpha)$ & $10^{-2}-10^{-4}$ \\
Steam temperature $\left(T_{s}\right)$ & $\leq 1000^{\circ} \mathrm{C}$ \\
Wall temperature $\left(T_{w}\right)$ & $300^{\circ} \mathrm{C}-1200^{\circ} \mathrm{C}$
\end{tabular}

different mechanistic models have been developed to simulate this flow [5], [6]. With this approach, different physical flow conditions can be simulated, since several correlations or models are coupled to the code for separate physical phenomena. Accordingly, the contributions of heat and mass transfer are estimated locally using closure models. Furthermore, we presented a mechanistic model implemented in a code-named NECTAR [1], to calculate heat and mass transfer phenomena and droplet dynamics in a polydispersed flow film boiling, at a sub-channel scale of a fuel assembly. The NECTAR code was validated thanks to the experimental data obtained from the COLIBRI experimental bank, under LOCA conditions in a low volume fraction of droplets $\left(\sim 10^{-4}\right)$.

In the present paper, modifications have been carried out in the COLIBRI bench, to increase the volume fraction of droplets in the experiments $\left(\sim 5.10^{-3}\right)$. Accordingly, this would affect the usual NECTAR calculation of heat transfer. In the first version of the code, we do not consider direct interaction between droplets in the flow or at the wall. For instance, as expressed by Castanet et. al [7], depending on the distance between the droplets, the steam-to-droplets convection could be reduced by more than $50 \%$. A similar effect is found for droplets' impact onto the wall, as in the case of a dense spray, droplets could interact at the wall, reducing the transfer area. Hence, to take this phenomenon into account, Breitenbach et al. [2] introduced the effective wetted substrate ratio $\left(n_{w e t}\right)$. Accordingly, this coefficient allows to estimate the reduction in the heat transfer generated by droplets interaction on the wall, as the measure of the probability of droplets interaction during the wall impact.

In this way, the present article aims to present the influence of the droplets interaction on the wall in the estimation of the heat removed by their impact using the NECTAR code. For this, Breitenbach's [2] effective wetted substrate ratio has been introduced in the usual NECTAR calculation. For this, NECTAR uses the correlation of Gradeck et al. [3] for the calculation of the heat flux by the impact of a drop and the estimation of Biance et al. [9] of the evolution of the diameter of a droplet during impact. For this analysis, the simulation was made for a straight tube with a droplets' volume fraction variation from $10^{-4}$ to 0.6 . 


\section{NECTAR code}

The code is based on the thermal balance between the heated wall and the internal flow of superheated vapor and dispersed drops. Figure 2 shows the different transfers: wall-tosteam convection $\left(\Phi_{w s}\right)$, wall-to-steam radiation $\left(\Phi_{r, w s}\right)$, steam-to-droplets convection $\left(\Phi_{s d}\right)$, steam-to-droplets radiation $\left(\Phi_{r, s d}\right)$, wall-to-droplets radiation $\left(\Phi_{r, w d}\right)$, wall-to-droplets direct impact $\left(\Phi_{i m p, w d}\right)$ and evaporation rate of droplets $\left(\dot{m}_{e v}\right)$. The model is $1 \mathrm{D}$ axial with two fluids (steam and droplets) in thermal and dynamic non-equilibrium. The droplets are spherical and considered to be saturated. There is no coalescence but the droplets fragmentation is possible. The wall is considered thermally "thin" $(\mathrm{Bi} \ll 1)$. Finally, the calculations are valid only during the Leidenfrost regime. Detailed information on the calculation of the different heat and mass transfer mechanisms as well as the dynamics of the droplets is given by Oliveira et al. [1].

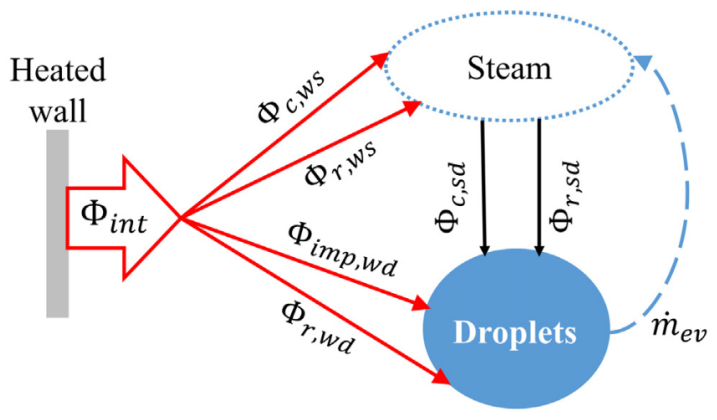

Figure 2. Heat transfers considered in NECTAR [1].

The heat flux dissipated from the wall due to the internal flow $\left(\Phi_{\text {int }}\right)$ is therefore the sum of four different contributions:

$$
\Phi_{i n t}=\Phi_{w s}+\Phi_{r, w s}+\Phi_{i m p, w d}+\Phi_{r . w d}
$$

The present work focuses on the characteristics of heat transfer by droplets impact onto the wall, i.e. $\Phi_{i m p, w d}$. Its calculation starts with the correlation proposed by Gradeck et al. [3], shown below, to estimate the heat transfer due to the impact of a single droplet onto a hot wall during the contact time $t_{R}$ :

$$
E_{1 d}=\int_{0}^{t_{R}} h_{w, s}\left(T_{w}-T_{s}\right) \pi R_{d}(t)^{2} d t+\int_{0}^{t_{R}} \varepsilon_{w} \sigma_{B}\left(T_{w}^{4}-T_{s a t}^{4}\right) \pi R_{d}(t)^{2} d t
$$

Where $\varepsilon_{w}, \sigma_{B}, T_{v}$ and $h_{w, s}$ are the wall surface emissivity, the Stephan-Boltzmann's constant, the film temperature of the steam, and the convective heat transfer coefficient at the wall, respectively. Furthermore, the experimental data obtained for $h_{w, s}$ by Gradeck et al [3] were used for the present study. Additionally, the contact time $t_{R}$ is estimated with the correlation of Rayleigh, see [8] with the modified coefficient of Biance et at [9], given as:

$$
t_{R}=2.65 \sqrt{\frac{\rho_{d} d^{3}}{8 \sigma_{t}}}
$$

$R_{d}(t)$ is the time function spreading diameter of the droplet. It was estimated from the analytical solution proposed by Gradeck et al [3] of the mass-spring-damper analogy described by Biance et al [9] to characterize the deformation of the droplet during impact. Thus, the following equation for the estimation of $R_{d}(t)$ was used:

$$
R_{d}(t)=\sqrt{2} R_{d}^{3 / 2}\left[\frac{g}{\omega_{0}^{2}} e^{-\lambda t}\left(\cos \left(\omega_{1} t\right)\right)-\frac{\omega_{0}}{\omega_{1}}\left(2 \pi r_{t}+r_{d}\right) \sin \left(\omega_{1} t\right)-\frac{g}{\omega_{0}^{2}}+d\right]^{-1 / 2}
$$


With $\lambda=\eta / m_{d}, \omega_{o}=\sqrt{k / m_{d}}, \omega_{1}=\sqrt{w_{o}^{2}-\lambda^{2}}, r_{d}=\lambda / \omega_{o}$ and $r_{t}=u_{n} \omega_{o} /(2 \pi g)$. In DFFB the droplets are polydisperse, so in order to take into account this distribution in the calculation, NECTAR code divides the probability distribution function $(P(d))$ into several bins, and for each section the mean Sauter diameter $d_{32, i}$ is calculated as:

$$
d_{32, i}=\frac{\int_{d_{i}}^{d_{i+1}} P(d) d^{3} \mathrm{~d} d}{\int_{d_{i}}^{d_{i+1}} P(d) d^{2} \mathrm{~d} d}
$$

With $d_{i}$, and $d_{i+1}$, the respective lower and upper limits of the bin $i$. Therefore, all the equations are solved for each diameter $d_{32, i}$, and the total rate of heat transfer for droplets impacts is the sum of the contributions of each bin. Finally, the heat flux $q_{i m p, w d}$ can be estimated as:

$$
q_{i m p, w d}=\sum_{i=1}^{N_{b i n s}} \frac{6 \dot{m}_{i}}{\pi \rho_{d} d_{32, i}^{3}} E_{1 d, i}
$$

Where $\dot{m}$ is the mass flux density of droplets impinging the wall. Moreover, NECTAR code estimates this mass flux with Hewitt et Govan [10] correlation for droplet deposition rate:

$$
\dot{m}=\left\{\begin{array}{cc}
\frac{0.18}{\sqrt{\rho_{s} D_{h} / \sigma_{t}}} C & \text { if } C / \rho_{s}<3 \\
\frac{0.083\left(C / \rho_{s}\right)^{-0.65}}{\sqrt{\rho_{s} D_{h} / \sigma_{t}}} C & \text { if } C / \rho_{s}>3
\end{array}\right.
$$

The estimation of the concentration of droplets in the steam core $C=\alpha \rho_{d}$ is calculated by the correlation used by Guo et Mishima [5]. Furthermore, the mass flux used for the calculation of each bin is defined as:

$$
\dot{m}_{i}=\dot{m}\left(d_{32, i}\right) \int_{d_{i}}^{d_{i+1}} P(d) \mathrm{d} d\left(d_{32, i}\right)
$$

The cooling of the wall by the impact of droplets could be affected by droplets interaction. This could occur in the case in which the spreading of a droplet during the impact is affected by the presence of a neighboring droplet. In this case, the exchange surface would decrease, affecting the total heat transfer of both droplets with the wall. Another case in which heat transfer can be affected is because of the decrease in the relative wetted area, where the heat transfer is major [2].

To take into account this effect, Breitenbach et al [2] defined an effective wetted substrate ratio $\eta_{\text {wet }}$, which accounts the reduction of the wetted area due to droplets interactions. This ratio is based on statistic analysis of impacts since the impacts of the droplets are completely random in space and time, and their impact follows the Poisson distribution [11]. Thus, the effective wetted substrate ratio is described as:

$$
\eta_{w e t}=\frac{1-e^{-\lambda}}{\lambda}
$$

$\lambda$ is the cumulative wetted area of the substrate by the spray, defined by:

$$
\lambda=\frac{2.1 \dot{m}}{\rho_{d} u_{n}}\left(1+0.36 W e^{0.48}\right)^{2}
$$


Where $W e$ and $u_{n}$ are the Weber number and the normal velocity of droplets. This droplet velocity normal to the wall can be approximately defined [5] as

$$
u_{n}=\frac{\dot{m}}{\rho_{d} \alpha}
$$

According to the definition of normal droplet velocity of Eq. 9, the cumulative wetted area of the substrate by the spray can be expressed as:

$$
\lambda=2.1 \alpha\left(1+0.36\left(\frac{\dot{m}^{2} d}{\rho_{d} \alpha^{2} \sigma_{t}}\right)^{0.48}\right)^{2}
$$

In this way, $\lambda$ increases its value (causing $\eta_{\text {wet }}$ to decrease) by increasing the value of the diameter of the droplets, volume fraction, and the droplets mass flux. Finally, the heat flux density can be obtained by the modification of the equation 5 :

$$
q_{i m p, w d}=\sum_{i=1}^{N_{b i n s}} \frac{6 \dot{m}_{i}}{\pi \rho_{d} d_{i}^{3}} E_{1 d, i} \eta_{w e t, i}
$$

\section{Results and discussion}

Numerical simulations were made with NECTAR to compare the cooling obtained in different cases. To do this, we used the following parameters:

- Wall temperature of $1000^{\circ} \mathrm{C}$ and steam temperature of $500^{\circ} \mathrm{C}$.

- Droplets' volume fraction between $1.10^{-4}$ to 0.6 .

- Tube hydraulic diameter of $D_{h}=11.78 \mathrm{~mm}$.

- And a droplets polydisperse distribution as shown in Fig. 3. With an average diameter $\left(d_{10}\right)$ of $400 \mu \mathrm{m}$, a maximum diameter of $1300 \mu \mathrm{m}$ and a minimum diameter of $5 \mu \mathrm{m}$.

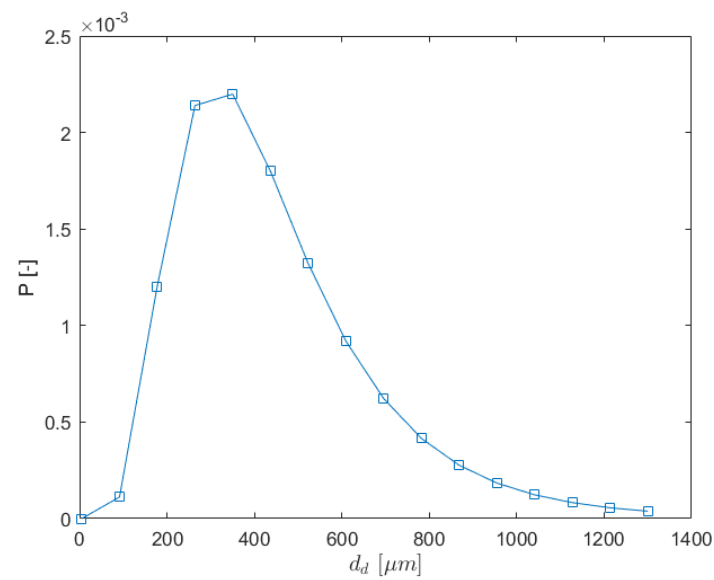

Figure 3. Log-normal distribution of droplets $\left(\mu=0.5, \sigma=\ln \left(d_{10}\right)\right)$.

The results of the heat flux by impact $q_{w, d}$ are represented in Fig. 4. The figure compares the calculation of the heat flux taking into account the interactions according to the Breitenbach ratio $\eta_{w e t}$ and without it (Eq. 11 and 5 respectively). As a result, the calculation of $q_{w, d}$ is 
not affected by droplets' interactions within the volume fraction range of a LOCA. Similarly, as shown in Fig. 5, droplet interactions have an almost negligible effect for small fraction volume droplet values. In such a way that for the droplets' volume fraction of $1.10^{-2}$ (the upper limit in LOCA condition), the decrease in heat flux is slightly above $1 \%$. This is because the mass flow of droplets hitting the wall is low, i.e., the probability that one droplet is affected by another at the moment of impact is also low in this case.

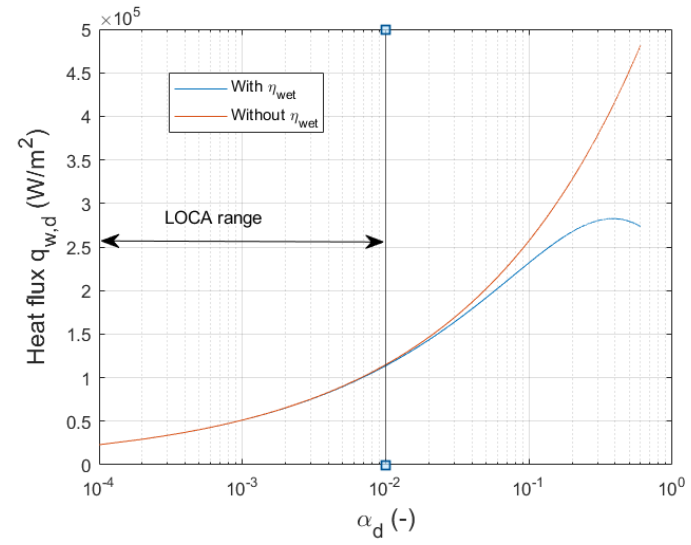

Figure 4. Heat flux of droplets impact into the wall in function of droplets' volume fraction.

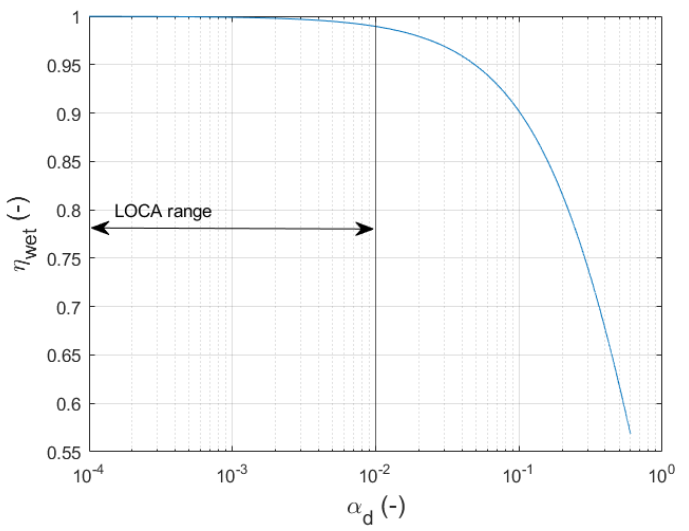

Figure 5. $\eta_{\text {wet }}$ in function of of droplets' volume fraction.

The droplet distribution used for the calculations covers the entire range of typical droplet diameters in a LOCA. On the other hand, the mass flux (according to Eq. 7) varies with the droplets volume fraction, the hydraulic diameter, and the steam density, since droplets are considered to be at saturated temperature. In this way, it is possible to increase the droplets' mass flux with a reduction in the hydraulic diameter and with an increase in steam temperature.

Consequently, in order to analyse the effect of the droplet interactions as a function of the mass of droplets impacting the wall, the following entry conditions were considered:

- Droplets mass flux between $0.1 \mathrm{~kg} / \mathrm{m}^{2} \mathrm{~s}$ and $10 \mathrm{~kg} / \mathrm{m}^{2} \mathrm{~s}$.

- Droplets volume fraction of $10^{-2}$.

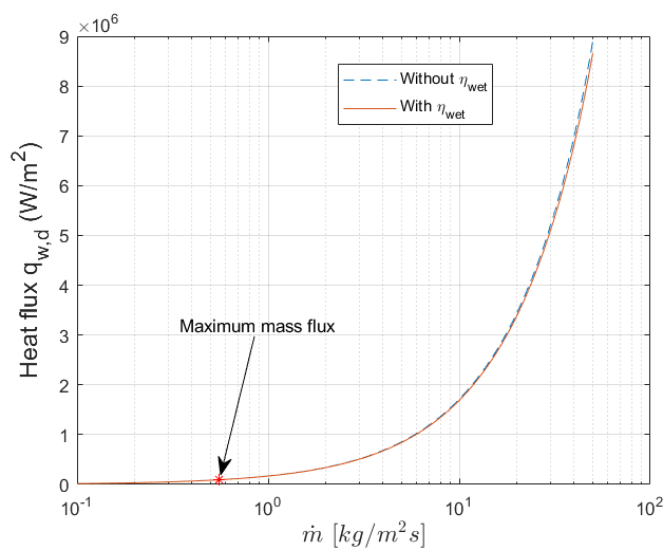

Figure 6. Heat flux of droplets impact into the wall in function of mass flux impining the wall.

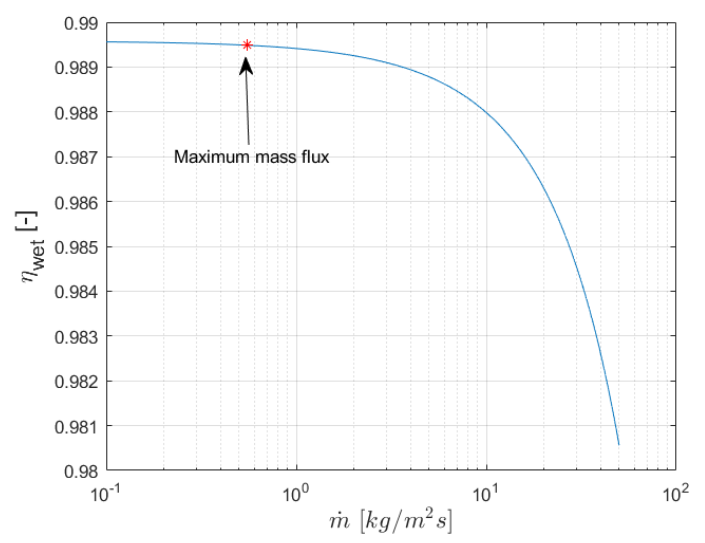

Figure 7. $\eta_{\text {wet }}$ as a function of mass flux impigning the wall.

Figure 6 and Fig. 7 represent the calculated flux with and without interactions and the values of $\eta_{\text {wet }}$, both as a function of different droplet mass flux values. Moreover, the corresponding maximum droplets' mass flux, in our case, is approximately $0.56 \mathrm{~kg} / \mathrm{m}^{2} \mathrm{~s}$. This is estimated 
with the Hewitt and Govan correlation (Eq. 6), with a steam temperature of $1000^{\circ} \mathrm{C}$ (maximum steam temperature) and with a hydraulic diameter of $3.72 \mathrm{~mm}$. This last value corresponds to the hydraulic diameter of a severely blocked subchannel [12].

Consequently, Fig.7 confirms that droplet interactions according to Breitenbach ratio $\eta_{w e t}$ seem to be negligible in LOCA conditions. Besides, in this case, $\eta_{\text {wet }}$ is less sensitive to variations in the mass flow of droplets that hit the wall than for variations in the droplets volume fraction.

\section{Conclusions}

The simulations carried out with NECTAR make it possible to analyze the influence of the droplets interactions on the thermal transfer by droplets impact which takes place in a tube representative of a PWR subchannel cooled by a steam/droplets flow during a LOCA. This analysis yielded the following results:

- Droplets interactions seems to be negligeble for the typical steam flow with dispered droplets in LOCA condition.

- A reduction of the passage area of the subchannel in the presence of ballooning fuel rods would not affect the usual calculation of heat transfer by impact of droplets. This is due to the fact that even under these conditions the droplets flux that hits the wall is low to be considered as a dense spray.

These results will be validated shortly from experimental data from the COLIBRI bench.

\section{Acknowledgements}

This work is carried out as part of a thesis funded by the french Institute for Radiation Protection and Nuclear Safety (IRSN) in Cadarache, France.

\section{Nomenclature}

$\begin{array}{ll}\text { Latin } & \text { letters } \\ d & \text { Droplet diameter [m] } \\ D_{h} & \text { Hydraulic diameter [m] } \\ E_{1 d} & \text { Energy single droplet [J] } \\ g & \left.\text { Gravity [m/ } \mathrm{s}^{2}\right] \\ h & \text { Heat transfer coefficient }\left[\mathrm{W} / \mathrm{m}^{2} \mathrm{~K}\right] \\ k & \text { Stiffness [N/m] } \\ m & \text { Mass [kg] } \\ \dot{m} & \text { Mass flux density }\left[\mathrm{kg} / \mathrm{m}^{2} \mathrm{~s}\right] \\ q & \left.\text { Heat flux [W/m }{ }^{2}\right] \\ R & \text { Radius [m] } \\ T & \text { Temperature [K] } \\ t & \text { Time [s] } \\ t_{R} & \text { Resident time [s] } \\ u & \text { Velocity [m/s] } \\ W e & \text { Weber number } \\ \text { Greek letters } \\ \alpha & \text { Volume fraction } \\ \eta & \text { Damping coefficient [Ns } / \mathrm{m}] \\ \eta_{w e t} & \text { Effective wetted substrate ratio } \\ \lambda & \text { Cumulative wetted area } \\ \mu & \text { Log-normal mean }\end{array}$

$\begin{array}{ll}\omega & \text { pulsation }[\mathrm{rad} / \mathrm{s}] \\ \Phi & \text { Rate of heat flow } \\ \rho & \text { Density }\left[\mathrm{kg} / \mathrm{m}^{3}\right] \\ \sigma & \text { Log-normal variance } \\ \sigma_{t} & \text { Surface tension }[\mathrm{N} / \mathrm{m}] \\ \sigma_{B} & \text { Stephan-Boltzmann constant } \\ \varepsilon & \text { Emissivity } \\ \text { Subscripts } \\ 0 & \text { initial state }(\mathrm{t} \leq 0) \\ 1 & \text { Final state }\left(\mathrm{t}>t_{R}\right) \\ d & \text { Droplet } \\ e v & \text { Evaporation } \\ h & \text { hydraulic } \\ i & \text { Bin class } \\ i m p & \text { Impact } \\ i n t & \text { Internal } \\ n & \text { Normal to the surface } \\ r & \text { Radiation } \\ s & \text { Steam } \\ s a t & \text { Saturation } \\ w & \text { Wall }\end{array}$




\section{References}

[1] A. V. S. Oliveira, J. D. Peña Carrillo, A. Labergue, T. Glantz, and M. Gradeck, "Mechanistic modeling of the thermal-hydraulics in polydispersed flow film boiling in LOCA conditions," Nucl. Eng. Des., vol. 357, no. August 2019, 2020, doi: 10.1016/j.nucengdes.2019.110388.

[2] J. Breitenbach, I. V. Roisman, and C. Tropea, "Heat transfer in the film boiling regime: Single drop impact and spray cooling," Int. J. Heat Mass Transf., vol. 110, pp. 34-42, 2017, doi: 10.1016/j.ijheatmasstransfer.2017.03.004.

[3] M. Gradeck, N. Seiler, P. Ruyer, and D. Maillet, "Heat transfer for Leidenfrost drops bouncing onto a hot surface," Exp. Therm. Fluid Sci., vol. 47, pp. 14-25, 2013, doi: 10.1016/j.expthermflusci.2012.10.023.

[4] M. J. Meholic, D. L. Aumiller, and F. B. Cheung, "A comprehensive, mechanistic heat transfer modeling package for dispersed flow film boiling - Part 1 - Development," Nucl. Eng. Des., vol. 291, pp. 295-301, 2015, doi: 10.1016/j.nucengdes.2015.07.013.

[5] Y. Guo and K. Mishima, "A non-equilibrium mechanistic heat transfer model for post-dryout dispersed flow regime," Exp. Therm. Fluid Sci., vol. 26, no. 6-7, pp. 861-869, 2002, doi: 10.1016/S0894-1777(02)00195-4.

[6] M. Andreani and G. Yadigaroglu, "Difficulties in modeling dispersed-flow film boiling," Wärme - und Stoffübertragung, vol. 27, no. 1, pp. 37-49, 1992, doi: 10.1007/BF01589976.

[7] G. Castanet, M. Lebouché, and F. Lemoine, "Heat and mass transfer of combusting monodisperse droplets in a linear stream," Int. J. Heat Mass Transf., vol. 48, no. 16, pp. 3261-3275, 2005, doi: 10.1016/j.jheatmasstransfer.2005.03.005.

[8] L. Bolle, J.C. Moureau, Spray cooling of hot surface, in: G.F. Hewitt, J.M. Delhaye, N. Zuber (Eds.), Multiphase Science and Technology, vol. 1, Hemisphere Publishing Ed., Washington, DC, 1986.

[9] A.L. Biance, F. Chevy, C. Clanet, G. Lagudeau, D. Quere, On the elasticity of an inertial liquid shock, J. Fluid. Mech. 554 (2006) 47-66.

[10] Hewitt, G. F., \& Govan, A. H. (1990). Phenomenological modelling of non-equilibrium flows with phase change. International Journal of Heat and Mass Transfer, 33(2), 229-242. https://doi.org/10.1016/0017-9310(90)90094-B

[11] W. Feller, An Introduction to Probability Theory and its Applications, third ed., vol. I, Wiley, New York, 1968.

[12] C. Grandjean, Coolability of blocked regions in a rod bundle after ballooning under LOCA conditions: Main findings from a review of past experimental programmes, Nuclear Engineering and Design 237 (15) (2007) 1872 - 1886, NURETH-11. doi:https://doi.org/10.1016/j.nucengdes.2007.02.022. 